\title{
VISER LA CONCILIATION EMPLOI-FAMILLE AU QUÉBEC : DES POLITIQUES POUR LES ENFANTS ET/OU LES MÈRES ?
}

\author{
Diane-Gabrielle Tremblay
}

CNAF | Informations sociales

\author{
2010/4 - $n^{\circ} 160$ \\ pages 106 à 113
}

\section{ISSN 0046-9459}

Article disponible en ligne à l'adresse:

http://www.cairn.info/revue-informations-sociales-2010-4-page-106.htm

Pour citer cet article :

Tremblay Diane-Gabrielle, « Viser la conciliation emploi-famille au Québec : des politiques pour les enfants et/ou les mères? ",

Informations sociales, 2010/4 $\mathrm{n}^{\circ} 160$, p. 106-113.

Distribution électronique Cairn.info pour CNAF.

(c) CNAF. Tous droits réservés pour tous pays.

La reproduction ou représentation de cet article, notamment par photocopie, n'est autorisée que dans les limites des conditions générales d'utilisation du site ou, le cas échéant, des conditions générales de la licence souscrite par votre établissement. Toute autre reproduction ou représentation, en tout ou partie, sous quelque forme et de quelque manière que ce soit, est interdite sauf accord préalable et écrit de l'éditeur, en dehors des cas prévus par la législation en vigueur en France. II est précisé que son stockage dans une base de données est également interdit. 


\section{Viser la conciliation emploi-famille au Québec : des politiques pour les enfants et/ou les mères?}

Diane-Gabrielle Tremblay - professeure

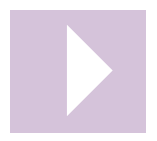

Se distinguant des autres régions du Canada et des États-Unis, le Québec a innové en matière de conciliation emploi-famille avec des politiques visant le bien-être des enfants autant que l'égalité des sexes. Multipliant par trois le nombre de places, I'instauration d'un réseau de garderies a préservé les droits des femmes sur le marché du travail et un nouveau régime de congé parental a renforcé les dispositions existantes et créé un congé paternel avec des semaines réservées au père.

Au cours des dernières décennies, le Québec a adopté plusieurs politiques sociales innovatrices qui visent la conciliation emploi-famille. Notamment, la ministre à l'origine de la création des centres à la petite enfance (services de garde) a mis en avant, au nom de l'égalité de droits entre les enfants, la contribution de cette politique à l'égalité en matière d'éducation de la petite enfance, quel que soit le milieu familial initial, mais aussi à l'égalité de droit d'accès au marché du travail pour les femmes ${ }^{(1)}$. En face de ceux qui étaient favorables à ce système de garde, certains (2) se sont inquiétés du « développement des enfants » en contexte de garderie, soutenant avec force que les mères devraient rester auprès de leur bébé au moins 18 mois, allant ainsi à l'encontre d'un objectif d'égalité des sexes. Le débat a duré plusieurs mois entre les deux camps. D’abord engagé sur le «bonheur éclairé de nos familles ", il a très vite opposé les partisans et les adversaires du système de garderies publiques au Québec. Pour ces derniers, les parents (les mères, précisait le docteur Chicoine dans certaines interventions) devraient rester auprès de leurs enfants pendant les premières années de vie, au risque de créer des problèmes de déficit affectif entre autres.

Au fil du temps, le débat est retombé et il semble bien que la majorité de la société au Québec considère que le service de garde public et à bas coûts est une excellente politique sociale, contribuant à la fois au bien-être des enfants et à l'égalité des droits des femmes. 


\section{Le Québec, une exception en Amérique du Nord}

À l'heure actuelle, le Québec se distingue des autres parties de l'Amérique du Nord - les États-Unis et le Canada anglais - en ce qui concerne ses choix en matière de conciliation et donc, plus largement, ses politiques sociales favorables aux enfants, et à leur famille, mais aussi aux droits des femmes. Pour mieux comprendre la situation particulière du Québec, nous exposerons ici l'essentiel des politiques en question, en nous attardant surtout sur les services de garde et sur le nouveau congé parental. Nous voulons aussi rappeler à cet égard le rôle joué par les acteurs sociaux : groupes de femmes, syndicats, ministère de la Famille, Conseil de la famille et de l'enfance et autres groupes d'intérêt (voir Tremblay, 2009).

Le Québec constitue en quelque sorte l'exception dans le contexte nordaméricain, du moins dans la mesure où le développement de ses politiques repose largement sur des modalités d'une gouvernance que nous avons qualifiée ailleurs d'inclusive (Tremblay, 2009). Ce type de gouvernance instaure un contexte au sein duquel les acteurs sociaux peuvent exprimer leurs points de vue sur les enjeux et sont en fait fréquemment invités par le législateur à le faire.

De plus, nous considérons que le Québec, en matière de relations travail-famille et de soutien à la parentalité, a adopté un modèle cumulatif (basé sur l'articulation travail-famille) alors que les États-Unis et le Canada anglais ont plutôt opté pour une attitude de laissez-faire qui, à ce jour, non seulement a limité les droits des travailleuses et travailleurs en matière de congés parentaux, mais aussi nié le principe de l'égalité de droits des enfants en ne soutenant pas financièrement la garde des jeunes enfants (3).

Ce sont pourtant deux aspects susceptibles d'avoir un impact sur le développement des enfants. Si certains soutiennent que les enfants seraient mieux servis en restant auprès de leur mère le plus longtemps possible, il semble bien que la majorité considère que les services de garde soutenus par l'État assurent un meilleur développement social des enfants, surtout de ceux de milieux défavorisés. Ces programmes encouragent aussi, bien sûr, la participation des femmes au marché du travail. Par ailleurs, on a observé que le nouveau congé parental québécois avait eu un impact sur le taux de fécondité (passé de 1,45 à 1,7 de 2006 à 2009). Au Canada anglais et aux États-Unis, c'est souvent la position inverse qui prévaut, à savoir que le bien-être de l'enfant prime sur les droits des femmes et que c'est aux familles d'assumer la charge des enfants.

\section{Les débats sur la parentalité}

Au cours des dernières décennies, le Québec a connu des débats importants autour des droits des enfants, des soins et de l'éducation qu'ils doivent 
recevoir dans la petite enfance, des enjeux de politique familiale et sociale, ainsi que de leur incidence sur l'égalité des sexes. Ceci est en grande partie imputable au rôle très actif que les associations familiales progressistes, les syndicats de travailleurs et particulièrement les groupes de femmes ont joué dans ce processus, ainsi que du livre évoqué plus haut (Collard et Chicoine, 2006) pour ce qui a trait à l'éducation du jeune enfant en particulier. Certains

\section{$\mathbf{6}$ L'accès universel à des services de garde à prix abordable a (...) occupé l'avant-plan de la politique familiale (...).}

groupes «familialistes » ont aussi soutenu cette vision, affirmant que les mères devraient rester à la maison pour s'occuper du jeune enfant, mais cette position est apparemment minoritaire au Québec.

Ainsi, avec en toile de fond ce questionnement sur l'intérêt pour l'enfant d'être gardé à domicile ou d'aller en garderie, les gouvernements successifs ont appuyé la position apparemment dominante visant à soutenir les services de garde. L'accès universel à des services de garde à prix abordable a donc occupé l'avant-plan de la politique familiale, avec la sécurité du revenu et les prestations de congé parental à tire de complément. Puis ce fut l'avènement du nouveau congé parental, visant une plus grande participation des pères et donc, non seulement l'égalité des droits des femmes sur le marché du travail, mais la reconnaissance du droit des pères à participer à la vie familiale et à l'éducation des enfants.

\section{Un nouveau congé parental qui encourage la participation des pères}

Depuis janvier 2006, le Régime québécois d'assurance parentale (RQAP) a remplacé les mesures du régime fédéral au Québec. Les nouvelles dispositions ne changent pas les celles prévues dans la loi sur les normes du travail du Québec, qui précisent la durée des congés de maternité (18 semaines) et du congé parental (52 semaines) pour une durée totale de 70 semaines sans salaire, tout comme les droits et obligations relatifs au départ et au retour au travail. Cependant, pour les personnes qui sont admissibles au régime parce qu'elles y ont contribué avec leur employeur, la loi prévoit des dispositions plus intéressantes pour les pères et les mères.

Le nouveau régime présente un certain nombre d'avantages eu égard à la population visée, à la flexibilité dans la prise du congé et au taux de remplacement du revenu. La dimension de la flexibilité dans la prise du congé résulte largement de débats sur l'importance de permettre une certaine flexibilité aux parents afin de mieux satisfaire leurs désirs du point de vue de la présence auprès du jeune enfant et sur le marché du travail.

D'importants débats avaient aussi eu cours concernant la présence du père, ou plutôt l'impact potentiellement négatif de l'absence du père auprès du jeune enfant, et les mouvements sociaux québécois ont revendiqué qu'une période de congé soit réservée au père et non transférable à la mère. Ainsi, 
selon l'expérience de l'Europe du Nord, où des semaines sont réservées au père (la Norvège et la Suède ont surtout été citées en exemple - voir Tremblay, 2008, et Haas et al., 2002), la présence du père le plus tôt et le plus longtemps possible a une incidence positive sur le développement de l'enfant. Dans ce contexte, le Québec a réservé une période de 3 à 5 semaines au père, avec l'objectif d'accroître sa participation en vue d'un meilleur développement du jeune enfant et ce par un temps réservé mais aussi par une hausse du taux de prestation et une plus grande flexibilité.

Il va sans dire que ces deux derniers éléments (hausse du taux de prestation et plus grande flexibilité dans les modalités d'usage du congé) visaient aussi à faciliter la participation des mères au congé et leur présence auprès de l'enfant. D'autres éléments visaient aussi à élargir cette présence, notamment l'inclusion des travailleurs indépendants parmi les bénéficiaires, les données ayant montré que, parmi ceux-ci, les mères retournent au travail lplus rapidement puisqu'elles ne bénéficient pas de prestations ou de congé de maternité. Le nouveau régime introduit plusieurs changements majeurs.

\section{Des semaines réservées au père}

Le premier changement est que les 3 ou 5 semaines réservées au père ne sont pas transférables à la mère, ce qui constitue une innovation au Canada et même en Amérique du Nord. Ce congé de paternité donne droit à des prestations plus élevées qu'avec le régime fédéral, puisque le taux de remplacement du revenu et le maximum de gains admissibles ont aussi été accrus, précisément afin de favoriser leur présence auprès des jeunes enfants. Le programme fédéral prévoyait un congé partageable entre le père et la mère, mais les données de Statistique Canada indiquent que cela n'a pas favorisé la participation des pères puisque les mères prenaient toujours 11 mois de congé en moyenne et qu'au maximum $14 \%$ des pères avaient pris une partie du congé entre 2001 et 2008.

\section{Augmentation du plafond de ressources}

Le deuxième changement est que le nouveau régime québécois augmente le revenu maximal assurable à $62500 \$$ dollars canadiens (environ 40000 euros en 2009) au lieu de 40000 \$, comme c’était le cas avec le congé fédéral.

\section{Assouplissement du régime}

Troisième innovation : les parents disposent maintenant de deux options, un régime de base (plus long mais moins rémunérateur), et un régime particulier ou spécifique (plus court mais plus rémunérateur). Ceci vise aussi à accroître le taux de participation au congé, et donc la présence auprès de l'enfant. Le financement de ce programme plus rémunérateur repose sur des cotisations supplémentaires que les employeurs, les employés et les travailleurs autonomes doivent verser au régime. Il y a bien sûr eu des débats, 
nombre d'employeurs et d'associations patronales s'opposant à verser ces nouvelles cotisations. Et, de fait, des débats autour de l'importance d'assurer un bon régime de congé parental afin d'assurer la plus grande présence possible auprès du jeune enfant et son meilleur développement dans le tout jeune âge, mais aussi l'égalité de droits des pères et des mères à participer à l'éducation et aux soins du jeune enfant. Si les employeurs étaient, en théorie, d'accord avec le fait qu'une présence du père auprès du tout jeune enfant est de la participation des pères au congé. bénéfique, ils arguaient de difficultés d'organisation et de coûts pour l'entreprise 9 pour refuser cette option. Finalement, la majeure partie de l'opinion publique et nombre de groupes syndicaux et associatifs, de femmes notamment, ayant longtemps défendu ces nouvelles options du congé parental, les gouvernements successifs (parti québécois, puis libéral) ont entériné ce congé réservé au père.

\section{Des résultats encourageants}

Peut-on évaluer l'impact du nouveau régime sur les pratiques des parents ? Depuis 2006, on a déjà observé une hausse de la participation des pères au congé. Plus de trois quarts des pères québécois prennent maintenant les 3 à 5 semaines de congé de paternité qui leur sont réservées, alors que dans le cadre du régime canadien, $15 \%$ environ des pères optent pour une partie du congé parental. En 2009, les pères québecois ont même pris, en moyenne, 7 semaines (Marshall, 2009). Pour cela, ils ont pris, en plus de leurs semaines réservées, une part du congé parental partageable entre la mère et le père. Le régime québécois d'assurance parentale a donc un effet important sur la présence auprès du jeune enfant, mais aussi sur la possibilité pour les mères d'être assurées de conserver leur emploi et de pouvoir y retourner après une maternité. Il permet de confirmer simultanément les droits des enfants et ceux des femmes. En outre, il contribue au changement des mentalités. Les nouvelles modalités ont induit des transformations importantes dans le monde du travail, où les pères affirment se sentir plus légitimes non seulement de prendre le congé parental mais aussi, comme il devient plus habituel de voir les pères s'absenter pour motifs familiaux, de prendre d'autres congés ou aménager leurs horaires pour motifs familiaux (Tremblay et Genin, 2008).

\section{Les services de garde pour l'égalité des femmes... et le bien-être des enfants}

Les services de garde sont le deuxième programme tout à fait important pour le développement et le bien-être des enfants, et la création du programme des Centres à la petite enfance (CPE) et leur financement par l'État ont généralement été justifiés par l'importance du rôle que peuvent jouer les CPE dans l'égalisation des chances des enfants, quel que soit leur 
milieu d'origine (voir Marois, in Bigras et Cantin, 2008). Il est important de souligner qu'entre 1992 et 2004, le Québec a augmenté les places en garderie de $310 \%$ alors que dans les neuf autres provinces du Canada, cette augmentation se chiffrait à $33 \%$ en moyenne (à l'autre extrême, Alberta a affiché une baisse de $7 \%$ ). (Doucet et al., 2009). L'importance accordée au développement de places en service de garde a

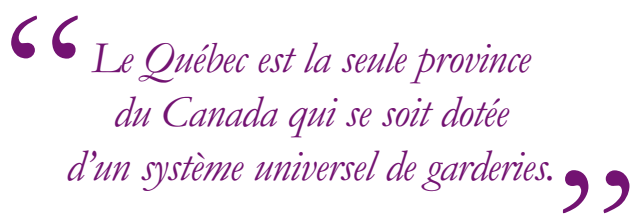
justement trait au fait que l'on considère que les CPE assurent une plus grande égalité de bien-être et de développement entre les enfants, pouvant ainsi compenser les inégalités dues au milieu familial différencié.

Le réseau québécois des CPE fut créé en 1997 sur les bases d'un réseau de centres existants, qui avaient été créés par des parents, des femmes et des collectivités dès les années 1970. En 1997, le réseau offrait 82000 places et il en comptait environ 200000 en 2008. Il manquait encore autour de 20000 places que le gouvernement du Québec a promis de créer entre 2008 et 2010, mais l'augmentation de la fécondité depuis la mise en place du nouveau congé parental pose à nouveau des défis sur ce plan. Pour compléter le réseau des CPE et ajouter des places plus rapidement, le gouvernement tente d'encourager le développement de places en installations privées ou dans des milieux familiaux : celles-ci (une femme qui garde jusqu’à 6 enfants à la maison) sont souvent préférées par les mères de plus jeunes enfants ou celles qui travaillent à horaires variables. L'extension des services vise à répondre à des besoins particuliers et ainsi à améliorer le bien-être de ces enfants, surtout les bébés.

Le Québec est la seule province du Canada qui se soit dotée d'un système universel de garderies. Les services sont abordables et de qualité généralement reconnue (en particulier dans le réseau public, car il y a aussi des établissements privés dont les services de garde sont en partie financés par l'État). Les autres provinces canadiennes n'ayant jamais reconnu l'importance de ce service dans le développement de l'enfant, aucun des neuf autres provinces et des trois territoires du Canada ne dispose de services comme ceux-ci.

La qualité de la garde des enfants y est non seulement insuffisante et inégale, mais aussi coûteuse, surtout si on la compare au Québec, la demande pour des services de garde dépassant largement l'offre des services privés. Bien que certains parents et groupes s'en plaignent, la vision dominante au Canada anglais comme aux États-Unis est que le privé peut très bien prendre ce type de service en charge, et l'incidence de l'inégalité de la qualité des services sur le développement des enfants ne fait apparemment pas débat, ou assez peu. Certaines évaluations indiquent qu'à l'extérieur du Québec les places en garderie peuvent à peine répondre à $20 \%$ de la demande pour les enfants de moins de 6 ans dont les parents travaillent (Doucet et al., 2009). Ainsi, outre 
le congé parental, les services de garde sont considérés comme un des éléments majeurs pour assurer une qualité d'éducation dès la prime enfance et assurer un développement psychologique et éducatif égal entre les enfants de diverses origines. Plusieurs études ont mis en évidence leur rôle majeur sur le développement des enfants (Bigras et Cantin, 2008 ; Robitaille, 2009). D'autres soulignent par exemple que la présence en garderie favorise le développement du langage, l'acceptation de la vie collective, l'habitude du partage et d'autres qualités chez les enfants.

Les paragraphes précédents donnent une idée de l'importante contribution des CPE, dans le modèle cumulatif ou de conciliation emploi-famille que l'on observe au Québec, à la politique sociale visant l'égalité de droits des enfants, dont le congé parental constitue un autre volet important. Dans le

66

(...) outre le congé parental, les services de garde sont considérés comme un des éléments majeurs pour assurer une qualité d'éducation dès la prime enfance et assurer un développement psychologique et éducatif égal entre les enfants de diverses origines. contexte québécois, ces deux mesures visent clairement l'égalité des droits de tous, tant ceux des jeunes enfants que ceux des pères et des mères de participer à la fois au marché du travail (Cette et al., 2009) et aux soins et à l'éducation des enfants.

Dans les autres juridictions du Canada, et surtout aux États-Unis, on considère généralement les politiques québécoises sur

les garderies et sur les congés parentaux, de même que les autres mesures destinées à améliorer l'équilibre emploi/famille comme une intervention excessive de l'État, alors que certains acteurs sociaux (syndicats et groupes de femmes en particulier) les voient plutôt comme des modèles. Au Québec, elles apparaissent comme des politiques sociales essentielles au soutien des droits des femmes, de la parentalité et de l'enfant.

\section{Notes}

1 - Voir I'article de Pauline Marois dans Bigras et Cantin (2008).

2 - Dont Nathalie Collard et le Dr Jean-François Chicoine (2006) dans leur livre, Le bébé et l'eau du bain 2006, Éditions Québec-Amérique.

3 - Voir les sites de l'Alliance de recherche université-communauté (Aruc) sur la gestion des âges et des temps sociaux (www.teluq.uqam.ca/aruc-gats) et de la chaire de recherche du Canada sur les enjeux socio-organisationnels de l'économie du savoir (www.teluq.uqam.ca/chaireecosavoir). 


\section{Bibliographie}

- Bigras N. et Cantin G. (dir.), 2008, Les services de garde éducatifs à la petite enfance du Québec. Recherches, réflexions et pratiques, Québec, Presses de I'Université du Québec.

- Cette G., Méda D., Sylvain A. et Tremblay D.-G., 2009, « Articulation entre les vies professionnelle et familiale. Une analyse des écarts entre la France et le Canada et un regard sur la conciliation », in Barrère-Maurisson M.-A. et Tremblay D.-G., (dir.) Concilier travail et famille. Le rôle des acteurs. Québec-France, Québec, Presses de l'Université du Québec, p. 129-167.

- Chicoine J.-F. et Collard N., 2006, « Le bébé et l'eau du bain »,Éd. QuébecAmérique.

- Doucet A., McKay L. et Tremblay D.-G., 2009, «Parental Leave in Canada and Québec : how to explain the different routes taken ? ", in Moss P. et Kamerman S. (dir.), The Politics of Parental Leave Policies, Bristol, Policy Press.

- Haas L., Allard K. et Hwang P., 2002, « The Impact of Organizational Culture on Men's Use of Parental Leave in Sweden », Community, Work \& Family, n 5, p. 319-341.

- Robitaille J., 2009, « Un portrait des institutions, du partenariat et des modes de garde éducative au Québec » in Barrère-Maurisson M.-A. et Tremblay D.-G. (dir.), Concilier travail et famille, Le rôle des acteurs, Québec-France, Québec, Presses de l'Université du Québec, p. 355-360.

- Tremblay D.-G., 2009, "La gouvernance de la conciliation emploi-famille au Québec. Le rôle des acteurs », in Barrère-Maurisson M.-A. et Tremblay D.-G. (dir.), 2009, Concilier travail et famille. Le rôle des acteurs. Québec-France. Québec, Presses de I'Université du Québec, p. 61-81;2008, « Les politiques familiales et I'articulation emploi-famille au Québec et au Canada », in Bigras N. et Cantin G., 2008, p. 13-26; Conciliation emploi-famille et temps sociaux, ( $2^{\mathrm{e}}$ édition)

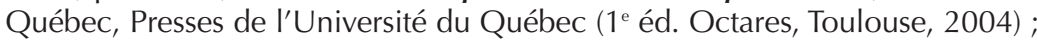
2003, "Articulation emploi-famille : Comment les pères voient-ils les choses?", Politiques sociales, Bruxelles et Madrid, vol. 63, n 3-4, automne, p. $70-86$.

- Tremblay D.-G. et Genin É., 2008, « Perceived Organisational Support to Work-Life Balance in a Municipal Police Service », rapport de recherche de la Chaire de recherche du Canada sur les enjeux socio-organisationnels de l'économie du savoir et de l'Alliance de recherche université-communauté sur la gestion des âges et des temps sociaux (CRC-Eses et Aruc-Gats), article à paraître dans International Journal of Sociology and Social Policy en 2010. 